Published in final edited form as:

Inflamm Bowel Dis. 2013 ; 19(4): . doi:10.1097/MIB.0b013e3182802a0a.

\title{
The overlap of functional abdominal pain in pediatric Crohn's disease
}

\author{
Lori A. Zimmerman, MD ${ }^{1}$, Arvind I. Srinath, MD $^{2}$, Alka Goyal, MD2, Athos Bousvaros, MD \\ MPH $^{1}$, Peter Ducharme, MSW ${ }^{4}$, Eva Szigethy, MD PhD ${ }^{3,}$, and Samuel Nurko, MD MPH ${ }^{1,{ }^{*}}$ \\ ${ }^{1}$ Department of Gastroenterology/Nutrition, Children's Hospital Boston \\ 2Department of Gastroenterology/Nutrition, Children's Hospital Pittsburgh \\ ${ }^{3}$ Department of Psychiatry, University of Pittsburgh, Children's Hospital Pittsburgh \\ ${ }^{4}$ Department of Psychiatry, Children's Hospital Boston
}

\begin{abstract}
Background-Children with Crohn's disease (CD) may report abdominal pain despite clinical remission, suggesting that functional abdominal pain (FAP) may be playing a role.
\end{abstract}

\begin{abstract}
Aim-This study aims to explore the presence and impact of FAP in children with CD in remission.
\end{abstract}

Methods-Children, aged 9-17, with CD were enrolled. Demographic information, the Pediatric Crohn's Disease Activity Index (PCDAI), and the Children's Depression Inventory (CDI) were obtained. Disease remission was defined by physician global assessment, normal labs, and absence of 3 or more stools a day, nocturnal stooling, bloody diarrhea, concurrent steroid therapy, strictures, or disease flare within 6 months. FAP was defined as patients with abdominal pain and CD remission. Rates of depression (CDI >9) were compared.

Results-139/307 children reported abdominal pain. Of this group, 18/139 (13\%) met criteria for FAP. Despite clinical remission, 8/18 CD FAP patients were classified with active disease by PCDAI. CD FAP patients had a higher rate of depression than CD patients in remission with no abdominal pain (55.6\% vs. 29.9\%; $\mathrm{p}=0.03$ ), similar to patients with abdominal pain from active CD (55.6\% vs. $44.8 \%$; $\mathrm{p}=0.62)$.

Conclusions-A proportion of children with CD in remission have FAP. These children are at significant risk for depression. Future studies are needed to determine whether depression contributes to functional pain development or if pain itself leads to depression. Especially given that functional pain may exaggerate disease activity, clinicians caring for children with $\mathrm{CD}$ and FAP should consider evaluating for depressive disorders before escalating therapy.

\section{Keywords}

Crohn's disease; Inflammatory Bowel Disease; Functional Abdominal Pain; Irritable Bowel Syndrome; Depression

Contact information for corresponding author: Samuel Nurko MD Children's Hospital Boston, Department of Gastroenterology and Nutrition, 300 Longwood Avenue, Boston MA 02115. samuel.nurko@ childrens.harvard.edu. Phone 617-355-6055. Fax 617-730-0495.

* Dr Nurko and Dr Szigethy contributed equally to the present manuscript

Publisher's Disclaimer: This is a PDF file of an unedited manuscript that has been accepted for publication. As a service to our customers we are providing this early version of the manuscript. The manuscript will undergo copyediting, typesetting, and review of the resulting proof before it is published in its final citable form. Please note that during the production process errors may be discovered which could affect the content, and all legal disclaimers that apply to the journal pertain. 


\section{Introduction}

Abdominal pain is a common complaint in children with Inflammatory Bowel Disease (IBD), which often indicates active disease and inflammation. However patients with Crohn's disease (CD) in remission may report varying degrees of gastrointestinal symptoms (e.g., bloating, diarrhea, urgency, constipation, and incomplete bowel movements) and abdominal pain, suggesting that functional gastrointestinal disorders, including functional abdominal pain (FAP), may be present.(1-5) Given that inflammation often correlates with pain, CD patients in apparent remission by labs, radiology, and endoscopy with ongoing pain present a diagnostic and therapeutic dilemma to practitioners. Evaluation of the pain can lead to unnecessary costs, invasive testing, and escalation of therapy when the underlying etiology is functional in nature rather than from active CD.

The overlap of symptoms in CD and functional bowel disorders are also evident in CD severity measures. Specifically, the Pediatric Crohn's Disease Activity Index (PCDAI), a tool used among clinicians and researchers to objectively categorize disease severity in pediatric CD patients (6), includes symptoms (e.g., abdominal pain and changes in stool consistency/frequency) commonly seen in both $\mathrm{CD}$ and functional bowel disorders. Thus, the presence of functional symptoms alone can affect disease severity scores for patients who may be in clinical remission and have ongoing functional symptoms.

Yet research on the impact of functional disorders on pediatric IBD is sparse. No pediatric studies, to our knowledge, have attempted to estimate the occurrence of FAP for symptomatic $\mathrm{CD}$ patients in clinical remission. In addition, research has yet to examine the effect of FAP on IBD severity categorization. Similarly, the influence of psychological factors, such as depression, on pain perception and coping in these patients is unclear.

The aims of the present study are (1) to demonstrate the occurrence of FAP in pediatric CD patients in remission; (2) to examine the effect of FAP on PCDAI categorization; and (3) to compare the characteristics and risk of depression in pediatric CD patients with FAP to other children with $\mathrm{CD}$. We hypothesized that a proportion of children with $\mathrm{CD}$ in remission have abdominal pain from functional disorders. The presence of functional abdominal pain is likely to overestimate CD activity and increase risk of depression in this group of patients.

\section{Material and Methods}

\section{Study Design}

The study cohort consisted of 307 patients, aged 9-17, with CD that were enrolled prospectively as a sub-study of a cognitive behavioral therapy trial taking place over 18 months at two collaborating centers, Children's Hospital Boston and Children's Hospital of Pittsburgh. This sub-study included all children with CD at the appropriate developmental level for behavioral therapy who were screened for depression regardless of pre-existing clinical suspicion. Patients were recruited from the outpatient clinics, inpatient floors, and infusion centers. CD was confirmed by endoscopy and histology. The Pediatric Crohn Disease Activity Index (PCDAI), a well-validated scale comprised of patient history, physical exam, laboratory, and growth parameters was used to assess pediatric CD severity. The Children's Depression Inventory (CDI)(7), a validated instrument using both child and parent report, was used to assess depressive symptoms in these subjects. Medical records were reviewed to obtain demographic information, and other variables of disease activity at screening, including clinical symptoms, changes in therapy, and laboratory, radiologic, and endoscopic results. Information on disease relapse was collected and defined by physician 
global assessment as supported by laboratory and gross endoscopic findings, when applicable.

\section{Definition of Terms}

As supported by the literature $(1,4)$, clinical disease remission was defined by the physician's global assessment of remission by the treating gastroenterologist at the time of clinical appointment and by objective clinical criteria. All patients in remission had normal labs [e.g. $\operatorname{ESR}(<10)$, albumin $(>3.5)$, and $C R P(<1.0)]$ as well as the absence of clinical signs or symptoms of active disease (e.g. three or less stools a day, no bloody diarrhea, no nocturnal stooling, no strictures, no concurrent steroid treatment). Remission criteria also required no escalation in medical therapy or clinical relapse for at least 6 months. No signs of active inflammation were present in the relevant investigations if performed in the last 6 months. Abdominal pain was defined by a pain rating on the PCDAI of 5 ("mild-brief, does not interfere with all activities) or 10 ("mod/severe: daily, longer-lasting, affects activities, nocturnal).

Functional abdominal pain (FAP) was diagnosed in CD patients with abdominal pain despite clinical remission as defined above. The presence of depressive symptoms was defined by self-reported CDI >9.(8)

\section{Statistical Analysis}

Demographic variables were compared between three groups: FAP patients (abdominal pain and inactive disease), patients with abdominal pain and active disease, and patients with inactive disease and no abdominal pain. Wilcoxon rank sum tests were used for continuous variables given the nonparametric distribution of data. Chi squared analyses or Fisher's exact tests were employed for binary and categorical variables. For CD patients with FAP, a modified PCDAI was calculated by removing the abdominal pain score; rates of remission by PCDAI $\leq 10$ were then recalculated using this modified PCDAI. For comparison, the modified PCDAI was also calculated for patients with abdominal pain and active CD. Fisher's exact test was used to compare the rates of remission using the total and modified PCDAI scores for both the FAP group and for the group with active CD and pain. The proportions of patients with FAP and depressive symptoms by CDI were calculated. Fisher's exact test was used to compare rates of depression in the three groups of patients: CD FAP patients; patients with abdominal pain and active disease; and patients with inactive disease and no abdominal pain. The present findings provide a power of $70 \%$ with an alpha of 0.05 to find a difference in the percentage of patients with depression when comparing patients in remission with or without abdominal pain.

\section{Ethical Considerations}

The present study was conducted with approval from the Institutional Review Boards at both institutions in January 2008. Written informed consent was obtained from subjects.

\section{Results \\ Study Population}

Of the $307 \mathrm{CD}$ patients enrolled, 139 reported abdominal pain. Demographic and clinical characteristics of our study population are detailed and compared in Table 1. There were no significant statistical differences in demographics across groups except for PCDAI score. Eighteen patients met our criteria for FAP. The distribution of patients by pain and disease activity is detailed in Figure 1. The FAP group constituted $13 \%$ of all patients with abdominal pain (18/139) and $6 \%$ of the total sample (18/307). The average length of follow- 
up of FAP patients was 26 months. Of the 18 FAP patients, $10(56 \%)$ had a colonoscopy or MRI imaging of abdomen within the past year.

\section{Effect of functional abdominal pain on PCDAI}

The mean PCDAI score for patients with FAP was 12.9 (range 5-25). 8/18 (44\%) of patients with $\mathrm{CD}$ and FAP were classified with active disease by PCDAI score $>10$. When the pain scores were removed from the PCDAI score, only 4/18 (22\%) were classified with active disease $(\mathrm{p}=0.02)$. By comparison, abdominal pain also affected disease severity categorization in patients with abdominal pain and active disease. (Figure 2) Evaluation of the sub-group of CD FAP patients who had an abdominal MRI or colonoscopy in the previous 12 months revealed a similar effect of PCDAI. In this group, 6/10 (60\%) met criteria for active disease by PCDAI $>10$. When the abdominal pain score was removed, only $3 / 10(30 \%)$ of patients met criteria for active disease.

\section{Relationship between functional abdominal pain and depression}

In the total study population, 118/307 (38\%) met criteria for depression. Ten of eighteen CD FAP patients had depressive symptoms (55\%). In contrast, only 47/157 (30\%) of patients with no abdominal pain and inactive disease by PCDAI $\triangle 10$ met criteria for depressive symptoms $(\mathrm{p}=0.03)$. The rate of depression in CD FAP patients was similar to CD patients with abdominal pain from active disease $(59 / 121$ or $49 \%$; $\mathrm{p}=0.62)$. (Figure 3 )

\section{Discussion}

This study shows that functional abdominal pain occurs in pediatric patients with quiescent CD. In addition, these patients have a higher risk of depressive symptoms than other CD patients with inactive disease. In fact, children with $\mathrm{CD}$ and abdominal pain seem to be at risk of depression regardless of disease activity. The present findings also demonstrate that the PCDAI is affected by functional symptoms and may overestimate disease activity in patients with CD FAP, which may lead to unnecessary evaluation and treatment.

In our sample, we found that $13 \%$ of pediatric IBD patients with abdominal pain have pain from overlapping functional disease. The occurrence of functional bowel disorders in pediatric IBD patients in remission has not been well characterized. While FAP was previously described by Faure et al. in 8 children with $\mathrm{CD}$, they did not provide prevalence data.(9) Adult studies have also reported that IBD and functional bowel disorders may overlap. Minderhoud et al. examined 107 adult IBD patients in clinical remission and reported a prevalence of Irritable Bowel Syndrome (IBS) by Rome II criteria in $31 \%$ of ulcerative colitis (UC) patients, $41 \%$ of Crohn's disease (CD) patients, and $7.6 \%$ of healthy controls.(2) In addition, Farrokhyar et al. reported an $82 \%$ prevalence of functional disorders (mostly anorectal and bowel disorders) in a sample of 149 IBD patients with inactive disease(5). And, Piche et al. noted a similar prevalence of $45.6 \%$ of IBS-like symptoms in patients with quiescent CD.(3) Therefore, adult studies suggest an increased risk of functional bowel disorders in patients with Inflammatory Bowel Disease. In contrast, our findings suggest a prevalence of FAP in children with inactive IBD closer to the prevalence of functional disorders in a general population of children and adolescents (10). While we suspect that the current study may have underestimated the prevalence of FAP in children with IBD, the study contributes to existing knowledge by demonstrating that FAP can also occur in organic disease. The understanding of this potential overlap has the potential to decrease testing, medication escalation, and cost when the etiology is functional rather than from a flare of the underlying disease. The current study is the first in children to provide evidence that functional disorders occur in children with IBD in remission and should be considered. 
The genesis of abdominal pain in patients with IBD in remission is likely similar to patients with other functional disorders with biological, psychological, and psychosocial contributors to pain generation and perception.(11-13) The present study highlights the importance of depression in patients with $\mathrm{CD}$ and abdominal pain. The CD FAP patients were more depressed than other CD patients in remission and as depressed as patients with active disease and abdominal pain. Previous studies have shown that anxiety and depression are prevalent in children with IBD. $(14,15)$ This psychological distress can influence coping ability, pain perception, and disease activity.(16) A recent study in adult patients with CD has demonstrated higher levels of depression compared to healthy controls, especially in those patients with high levels of perceived stress. (17) The present study supports the significant risk of depression in children with $\mathrm{CD}$. These findings correlate depression to abdominal pain, showing higher rates of depression in children with Crohn's disease with abdominal pain regardless of disease activity. Still, our study cannot establish the causal link between depression and pain. That is, we cannot determine if the pain produced depression or if the presence of depression exacerbated the pain. Given that Faure et al. demonstrated with the use of barostat that pediatric CD patients in remission with pain had visceral hypersensitivity (9), it is possible that the pain is amplified by the depression. Independent of the causal relationship, the present study clearly shows that the presence of abdominal pain, whether in the context of active or inactive disease, is associated with almost double the rates of depressive symptoms compared to pediatric IBD patients without pain. Therefore, further studies to understand this link are needed.

Overall, anxiety and depression are also common in patients with functional gastrointestinal illness and may affect illness severity and extent of disease.(18) Adults with IBD and overlapping IBS-like symptoms have higher levels of anxiety, depression, and poor wellbeing than IBD patients without IBS. $(1,2)$ The overlap of functional disorders in patients with IBD has been shown to impact healthcare utilization and quality of life.(5) Piche et al. reported that $\mathrm{CD}$ patients in remission with functional disorders have higher levels of fatigue and depression than CD patients without functional disorders and similar to patients with IBS alone.(3) While pediatric data are limited, Gold et al. compared children with IBD to children with IBS and found that the children with IBS suffered more depression and worse quality of life than the children with IBD.(19) Previous research supports that children with frequent abdominal pain are at increased risk for depressive symptoms.(20) Thus, it is important to recognize the presence of functional disorders and depression both to provide appropriate treatment and psychological support and to avoid more invasive testing and escalation of immunosuppressive and biologic therapies with concomitant toxicities. On the other hand, if an increase in therapy is being contemplated for a Crohn's patient with abdominal pain, it is important to document evidence of inflammation to avoid over-treating patients with functional disease or psychological comorbidities.

One of the main difficulties in this area of research is that there is often an overlap of symptoms between active CD and FAP, which makes it difficult to exclude active disease. For this reason, we employed rigorous remission criteria compared to the criteria used in previous studies to better isolate the symptoms associated with purely functional disorders, even if it led to a potential under-estimation of FAP in this population. Specifically, we defined remission with subjective measures (e.g. the physician's global assessment) and objective measures (e.g. laboratory parameters and endoscopic and radiologic information when available). We relied on the treating gastroenterologist's expertise to evaluate for other potential causes of abdominal pain (e.g biliary colic) when appropriate. To include more information about disease course, we also incorporated information on the clinical evolution of patients and excluded patients with disease relapse or escalation of medical therapy within 6 months. Few studies have attempted to define this population so critically. 
However, the diagnosis of a functional disorder in the setting of IBD is also complex because Rome III criteria for FAP or IBS negate the possibility of underlying organic disease. While it can be argued that the Rome III criteria can be applied to IBD patients in remission, no prospective studies have validated the use of Rome III criteria in this population. The concept that functional disorders can also be present in patients with underlying organic disease is an emerging one. Clinical experience shows that it undoubtedly exists, but future studies are needed to establish the best way to make this diagnosis, which will have a major impact in the cost of patient evaluation and approach to treatment.

In an attempt to address how the presence of functional pain affects the perception of disease severity, we explored the effect of FAP on pediatric CD severity measures. Since abdominal pain is a major component of CD scoring tools, we investigated if using a modified PCDAI without an indicator for abdominal pain would change the categorization of disease activity. We hypothesized that the use of disease scoring tools would exaggerate disease activity in patients with an overlap of CD and FAP. In support of this hypothesis, almost half of our CD patients in remission with FAP met criteria for active disease by total PCDAI, but only $22 \%$ of these patients met criteria after removal of the pain score. However, since abdominal pain is also a symptom of active disease, the removal of the pain score also affected disease categorization in active patients. For this reason, we do not recommend a modification of the PCDAI without the abdominal pain score, as this would likely affect validation.

A recent adult study highlighted this difficulty in defining a population of patients with an overlap of IBD and functional disorders. Keohane measured a higher prevalence of IBS in IBD patients than the current study, but similar to previously reported adult studies using the Rome II criteria (59.7\% CD patients and 38.6\% UC patients). However, Keohane also found an increased level of fecal calprotectin in the patients defined with IBD-IBS overlap, suggesting that the symptoms were from occult inflammation rather than functional disorders.(4) This interpretation has been debated in the literature, as the role that intestinal inflammation may play in functional gastrointestinal disease is controversial. A unifying biopsychosocial model incorporating inflammation, cytokine activity, and/or HPA axis function with psychological distress and differences in central pain processing may better explain these patients' symptoms.(21) An alternative explanation for the elevated inflammatory markers in patients with functional bowel disorders is further supported by a pediatric study by Shulman et al. which demonstrated elevated fecal calprotectin in children with IBS or functional abdominal pain compared to healthy controls.(22) This further highlights the importance that inflammation may have in the pathophysiology of functional disorders. A prospective study using a reliable noninvasive biomarker is needed to better evaluate the prevalence of functional bowel disorders in pediatric $\mathrm{CD}$.

The current study has some limitations. We had to rely on existing clinical data in the definition of disease remission. Because not all patients underwent radiologic or endoscopic exploration, we could not include these criteria in our definition of disease remission. We also did not have information on fecal biomarkers, which have been validated to assess inflammation (e.g. fecal calprotectin or lactoferrin).(23-27) In practice, pediatric gastroenterologists often face similar clinical dilemmas when caring for CD patients with normal laboratory studies and stooling patterns but ongoing complaints of abdominal pain. The diagnostic and therapeutic yield of further exploration or medication escalation must be tempered with the risk of radiation, anesthesia, and toxicities of medications. Also, other studies that have attempted to define the prevalence of functional disorders in CD have used similar clinical data. 
Another limitation of the present study is its design, which prevented us from reinterviewing patients to more clearly establish their types of functional disorders. By Rome III criteria, patients with childhood functional abdominal pain disorder must have abdominal pain at least once a week for at least two months and some loss of daily functioning or other somatic complaints.(28) We were unable to define our population with Rome III standards as this information was not uniformly present in the medical records. Although it is controversial to apply Rome III criteria to patients with organic disease, a prospective study using Rome III criteria would better characterize the functional disorders present in these patients.

In conclusion we found that a proportion of patients with pediatric $\mathrm{CD}$ in remission have FAP. These patients are at greater risk for depression than other CD patients in remission. In fact, abdominal pain may be a risk factor for depression in children with CD independent of disease activity. Future studies will be needed to determine whether depression contributes to functional pain development in susceptible CD patients or if pain itself leads to depression. Clinicians caring for children with $C D$ and overlapping functional pain should consider evaluating for depression before escalating therapy. Also, the significant effect of pain on PCDAI categorization suggests that the PCDAI may overestimate disease activity in pediatric patients with overlapping $\mathrm{CD}$ and functional bowel disorders. A noninvasive biomarker is needed to better identify the patients with $\mathrm{CD}$ and functional disorders.

\section{Acknowledgments}

Financial support: This work was supported by NIMH R01 (R01MH07777) (Eva Szigethy), NIH K-24 (DK082792A) (Samuel Nurko)

\section{References}

1. Simren M, Axelsson J, Gillberg R, et al. Quality of life in inflammatory bowel disease in remission: the impact of IBS-like symptoms and associated psychological factors. Am J Gastroenterol. 2002; 97:389-396. [PubMed: 11866278]

2. Minderhoud IM, Oldenburg B, Wismeijer JA, et al. IBS-like symptoms in patients with inflammatory bowel disease in remission; relationships with quality of life and coping behavior. Dig Dis Sci. 2004; 49:469-474. [PubMed: 15139501]

3. Piche T, Ducrotte P, Sabate JM, et al. Impact of functional bowel symptoms on quality of life and fatigue in quiescent Crohn disease and irritable bowel syndrome. Neurogastroenterol Motil. 2010; 22:626-e174. [PubMed: 20403099]

4. Keohane J, O'Mahony C, O'Mahony L, et al. Irritable bowel syndrome-type symptoms in patients with inflammatory bowel disease: a real association or reflection of occult inflammation? Am J Gastroenterol. 2010; 105:1788, 1789-1794. quiz 1795. [PubMed: 20389294]

5. Farrokhyar F, Marshall JK, Easterbrook B, et al. Functional gastrointestinal disorders and mood disorders in patients with inactive inflammatory bowel disease: prevalence and impact on health. Inflamm Bowel Dis. 2006; 12:38-46. [PubMed: 16374257]

6. Hyams JS, Ferry GD, Mandel FS, et al. Development and validation of a pediatric Crohn's disease activity index. J Pediatr Gastroenterol Nutr. 1991; 12:439-447. [PubMed: 1678008]

7. Kovacs M. The Children's Depression, Inventory (CDI). Psychopharmacol Bull. 1985; 21:995-998. [PubMed: 4089116]

8. Szigethy E, Levy-Warren A, Whitton S, et al. Depressive symptoms and inflammatory bowel disease in children and adolescents: a cross-sectional study. J Pediatr Gastroenterol Nutr. 2004; 39:395-403. [PubMed: 15448431]

9. Faure C, Giguere L. Functional gastrointestinal disorders and visceral hypersensitivity in children and adolescents suffering from Crohn's disease. Inflamm Bowel Dis. 2008; 14:1569-1574.

[PubMed: 18521915] 
10. Chitkara DK, Rawat DJ, Talley NJ. The epidemiology of childhood recurrent abdominal pain in Western countries: a systematic review. Am J Gastroenterol. 2005; 100:1868-1875. [PubMed: 16086724]

11. Drossman DA, Whitehead WE, Toner BB, et al. What determines severity among patients with painful functional bowel disorders? Am J Gastroenterol. 2000; 95:974-980. [PubMed: 10763947]

12. Drossman DA. Presidential address: Gastrointestinal illness and the biopsychosocial model. Psychosom Med. 1998; 60:258-267. [PubMed: 9625212]

13. Grover M, Herfarth H, Drossman DA. The functional-organic dichotomy: postinfectious irritable bowel syndrome and inflammatory bowel disease-irritable bowel syndrome. Clin Gastroenterol Hepatol. 2009; 7:48-53. [PubMed: 18848909]

14. Szigethy E, Craig AE, Iobst EA, et al. Profile of depression in adolescents with inflammatory bowel disease: implications for treatment. Inflamm Bowel Dis. 2009; 15:69-74. [PubMed: 18831071]

15. Moser G. Depression and anxiety in inflammatory bowel disease. Gastroenterol Hepatol. 2009; 32(Suppl 2):9-12. [PubMed: 19900622]

16. Mawdsley JE, Rampton DS. Psychological stress in IBD: new insights into pathogenic and therapeutic implications. Gut. 2005; 54:1481-1491. [PubMed: 16162953]

17. Goodhand JR, Wahed M, Mawdsley JE, et al. Mood disorders in inflammatory bowel disease: Relation to diagnosis, disease activity, perceived stress, and other factors. Inflamm Bowel Dis. 2012

18. Bennett EJ, Piesse C, Palmer K, et al. Functional gastrointestinal disorders: psychological, social, and somatic features. Gut. 1998; 42:414-420. [PubMed: 9577351]

19. Gold N, Issenman R, Roberts J, et al. Well-adjusted children: an alternate view of children with inflammatory bowel disease and functional gastrointestinal complaints. Inflamm Bowel Dis. 2000; 6:1-7. [PubMed: 10701143]

20. Youssef NN, Atienza K, Langseder AL, et al. Chronic abdominal pain and depressive symptoms: analysis of the national longitudinal study of adolescent health. Clin Gastroenterol Hepatol. 2008; 6:329-332. [PubMed: 18258491]

21. Long MD, Drossman DA. Inflammatory bowel disease, irritable bowel syndrome, or what?: A challenge to the functional-organic dichotomy. Am J Gastroenterol. 2010; 105:1796-1798. [PubMed: 20686466]

22. Shulman RJ, Eakin MN, Czyzewski DI, et al. Increased gastrointestinal permeability and gut inflammation in children with functional abdominal pain and irritable bowel syndrome. J Pediatr. 2008; 153:646-650. [PubMed: 18538790]

23. Diamanti A, Colistro F, Basso MS, et al. Clinical role of calprotectin assay in determining histological relapses in children affected by inflammatory bowel diseases. Inflamm Bowel Dis. 2008; 14:1229-1235. [PubMed: 18398894]

24. Fagerberg UL, Loof L, Lindholm J, et al. Fecal calprotectin: a quantitative marker of colonic inflammation in children with inflammatory bowel disease. J Pediatr Gastroenterol Nutr. 2007; 45:414-420. [PubMed: 18030206]

25. Walkiewicz D, Werlin SL, Fish D, et al. Fecal calprotectin is useful in predicting disease relapse in pediatric inflammatory bowel disease. Inflamm Bowel Dis. 2008; 14:669-673. [PubMed: 18240279]

26. Foell D, Wittkowski H, Roth J. Monitoring disease activity by stool analyses: from occult blood to molecular markers of intestinal inflammation and damage. Gut. 2009; 58:859-868. [PubMed: 19136508]

27. Kane SV, Sandborn WJ, Rufo PA, et al. Fecal lactoferrin is a sensitive and specific marker in identifying intestinal inflammation. Am J Gastroenterol. 2003; 98:1309-1314. [PubMed: 12818275]

28. Rasquin A, Di Lorenzo C, Forbes D, et al. Childhood functional gastrointestinal disorders: child/ adolescent. Gastroenterology. 2006; 130:1527-1537. [PubMed: 16678566] 


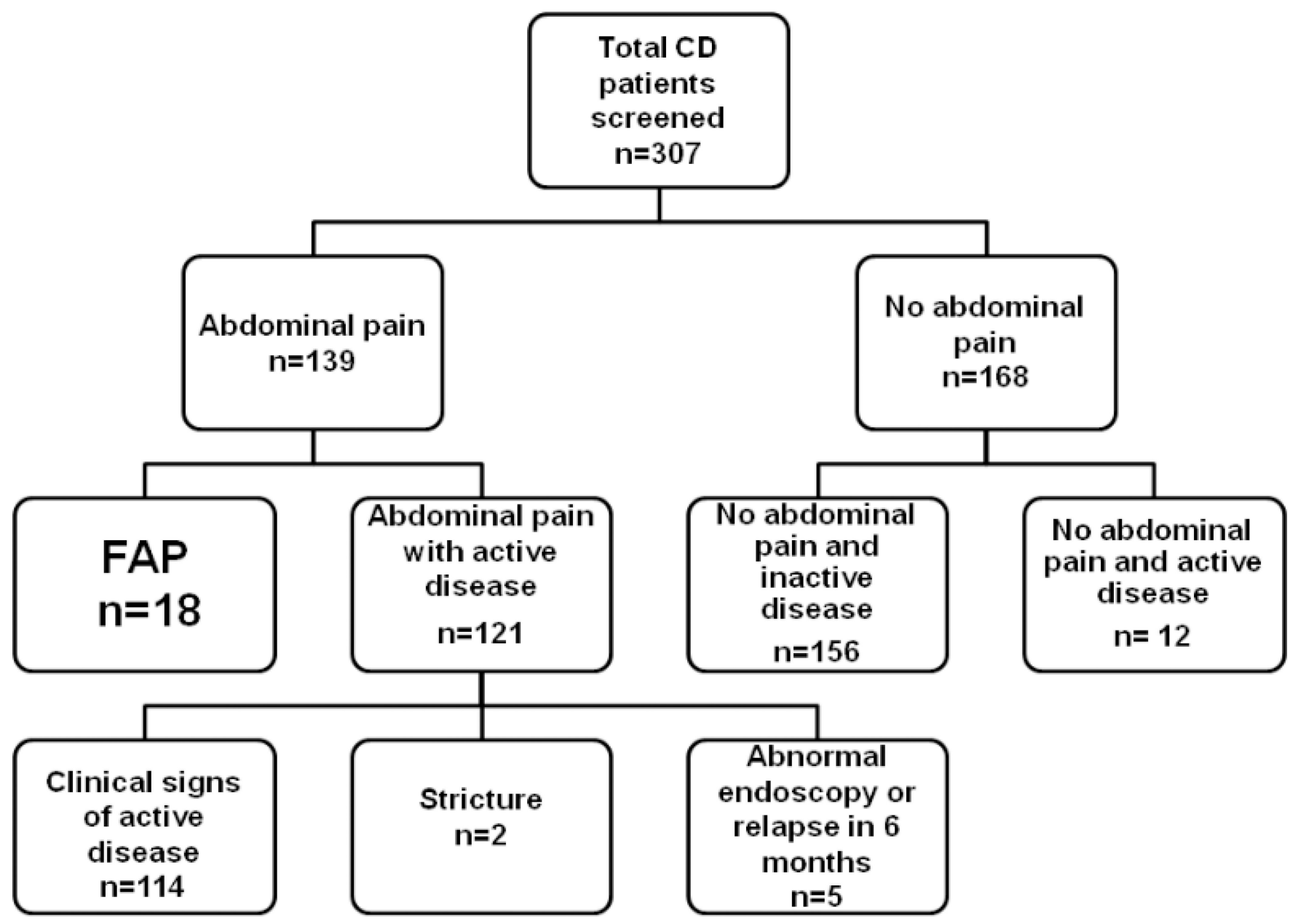

Figure 1. Classification of patients by presence of abdominal pain and disease activity FAP patients are defined as patients with abdominal pain but no active disease.

*Clinical signs and symptoms of active disease included abnormal labs $[E S R(<10)$, albumin (>3.5), and $C R P(<1.0)$ ], three or more stools a day; bloody diarrhea, nocturnal stooling, and the need for current steroid treatment. 


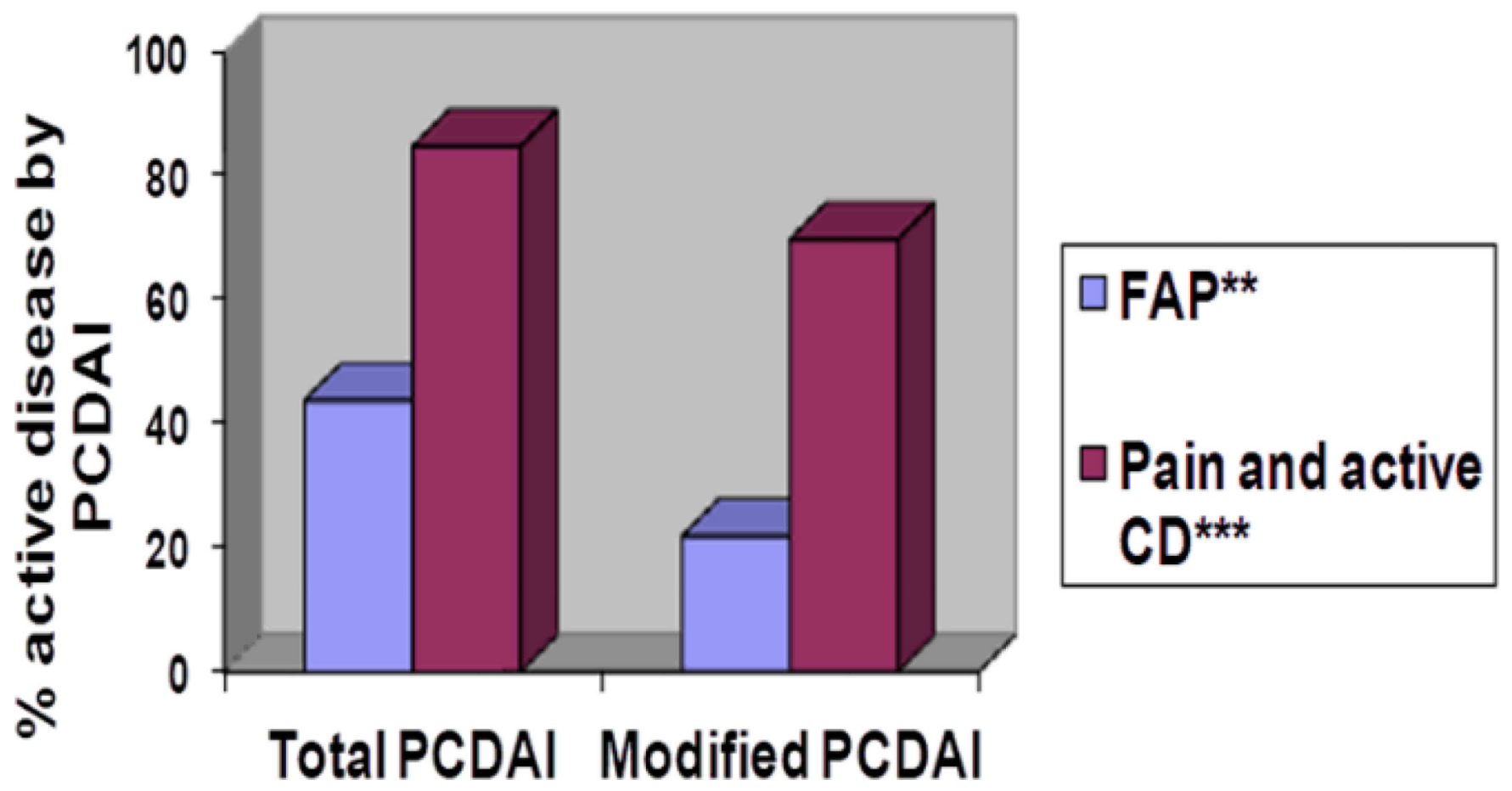

Figure 2. Difference in proportion of patients categorized with active disease* by total PCDAI and by modified PCDAI

*Active disease categorization is defined by PCDAI $>10$. In the modified PCDAI, the abdominal pain score is removed.; ** $\mathrm{p}=0.02 ; * * * \mathrm{p}=0.003$ 


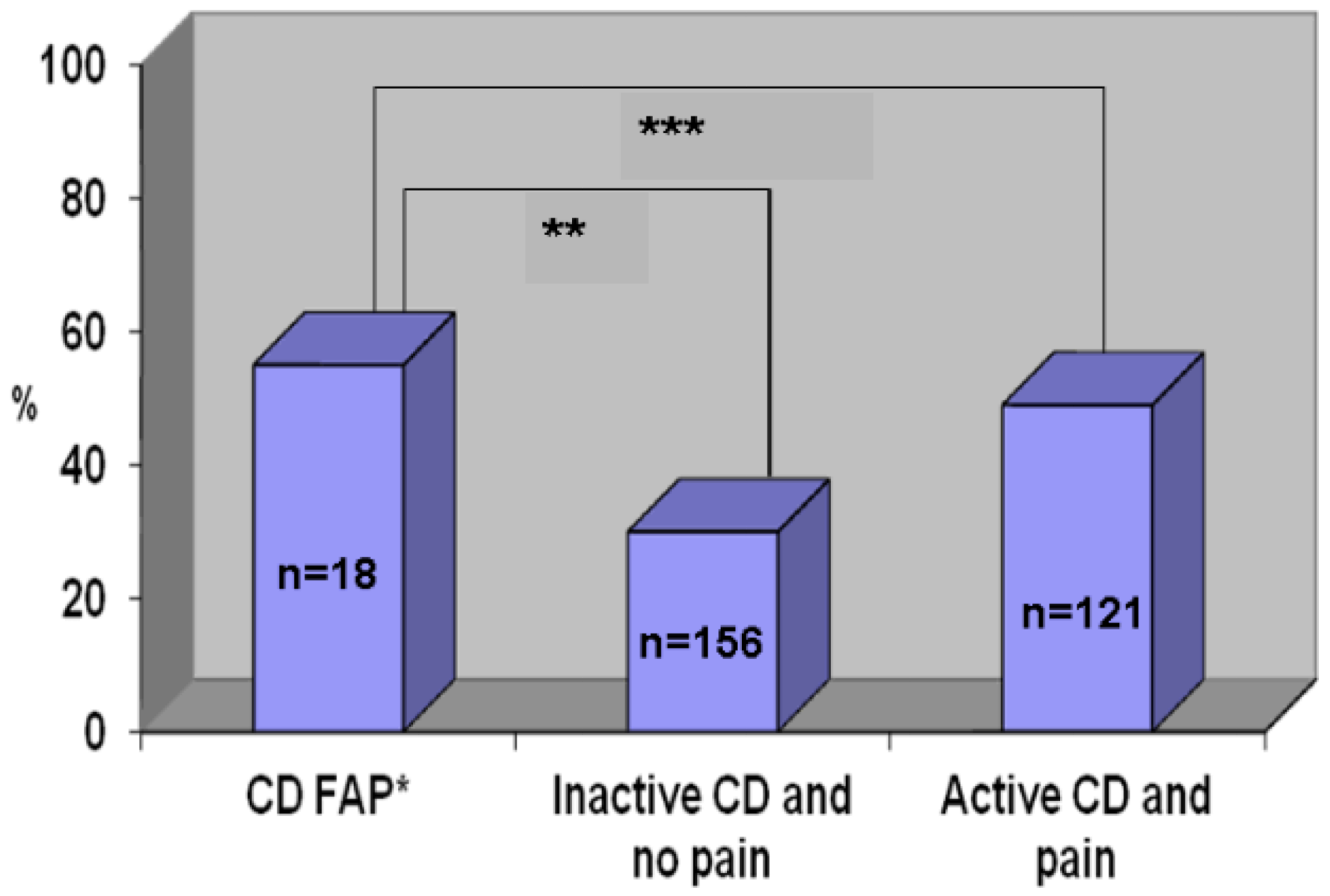

Figure 3. Proportion of CD FAP patients with depressive symptoms* compared to other CD patients

*Depressive symptoms defined by CDI $>9 ; * * \mathrm{p}=0.03 ; * * * \mathrm{p}=0.62$ 


\section{Table 1}

Demographic Information of CD patients with FAP, CD with active disease and pain, and CD with inactive disease and no pain

\begin{tabular}{|c|c|c|c|c|}
\hline & $\begin{array}{c}\text { FAP } \\
\text { patients } \\
\mathbf{N = 1 8}\end{array}$ & $\begin{array}{c}\text { Abdominal pain } \\
\text { and active disease } \\
\mathbf{N = 1 2 1}\end{array}$ & $\begin{array}{c}\text { No abdominal } \\
\text { pain and inactive } \\
\text { disease N=156 }\end{array}$ & P value \\
\hline Median age in years (IQR) & $13(11,16)$ & $14(12,16)$ & $15(13,16)$ & 0.489 \\
\hline Female n(\%) & $10(56)$ & $73(60)$ & $72(46)$ & 0.062 \\
\hline White n(\%) & $17(94)$ & $105(88)$ & $140(91)$ & 0.804 \\
\hline Median PCDAI (IQR) & $10(10,15)$ & $26(15,38)$ & $5(0,10)$ & 0.000 \\
\hline
\end{tabular}

